\title{
Necesidades de formación en investigación de profesores en ejercicio ${ }^{1}$
}

\author{
Brigitte Johana Sánchez Robayo² \\ Jaime Fonseca González ${ }^{3}$ \\ José Torres Duarte 4 \\ Jorge Rodríguez Bejarano ${ }^{5}$
}

Fecha de recepción: 26 de abril de 2015

Fecha de revisión: 14 de mayo de 2015

Fecha de aprobación: 30 de mayo de 2015

\section{Resumen}

1 El tipo de artículo es una investigación científica y tecnológica. Expone resultados del proyecto de investigación "Formación en y hacia la investigación de profesores de matemáticas", financiado por Colciencias y la Universidad Distrital Francisco José de Caldas.

2 Magister en Docencia de las Matemáticas. Docente de planta de la Universidad Distrital Francisco José de Caldas en el proyecto curricular de Licenciatura en Educación Básica con Énfasis en Matemáticas, sede la Macarena, carrera 3 No. 26A-40, oficina coordinación LEBEM, teléfono de contacto: 3237300 ext. 6340. Miembro de los grupos de Investigación Crisálida y EDUTOPÍA, en la línea de investigación de Formación de Profesores y Educación Matemática Crítica. Correo electrónico: bjsanchezr@udistrital.edu.co

3 Magister en Docencia de las Matemáticas. Docente de la Universidad Distrital Francisco José de Caldas en el proyecto curricular de Licenciatura en Educación Básica con Énfasis en Matemáticas, ubicado en la sede la Macarena, carrera 3 No. 26A-40, oficina coordinación LEBEM, teléfono de contacto: 3237300 ext. 6340. Miembro del grupo de investigación Crisálida, en la línea de investigación de Formación de Profesores. Correo electrónico: jaimejaimef@hotmail.com

4 Magister en Docencia. Docente de planta de la Universidad Distrital Francisco José de Caldas en el proyecto curricular de Licenciatura en Educación Básica con Énfasis en Matemáticas, sede la Macarena, carrera 3 No. 26A-40, oficina coordinación LEBEM, teléfono de contacto: 3237300 ext. 6340. Miembro de los grupos de investigación Crisálida y EDUTOPÍA, en la línea de investigación de Formación de Profesores y Educación Matemática Crítica. Correo electrónico: jotorresd@udistrital.edu.co

5 Magister en Educación. Docente de planta de la Universidad Distrital Francisco José de Caldas en el proyecto curricular de Licenciatura en Educación Básica con Énfasis en Matemáticas, sede la Macarena, carrera 3 No. 26A-40, oficina coordinación LEBEM, teléfono de contacto: 3237300 ext. 6340. Miembro del grupo de investigación Crisálida, en las líneas de investigación de Transición aritmética-álgebra y Calidad de la Educación. Correo electrónico: jorgeedumat@udistrital.edu.co 
Investigar la práctica favorece la reflexión sobre el ejercicio profesional de los profesores, aporta elementos para construir conocimiento profesional, permite constituir comunidades interesadas en problematizar la práctica y los procesos de enseñanza-aprendizaje de las matemáticas. Así, la formación en investigación se convierte en una herramienta para pensar y transformar prácticas educativas escolares y construir conocimiento profesional docente. Como insumo para diseñar una propuesta de formación en investigación para profesores de matemáticas en ejercicio, se realizó un estudio en el que se identificaron necesidades de formación en investigación mediante ocho estudios de caso, empleando la observación no participante

y la entrevista como instrumentos de recolección de información, considerando como fuentes de información a profesores de matemáticas, sus compañeros y directivos o coordinadores. Las principales necesidades detectadas se asocian con las dinámicas de trabajo en grupo, la socialización de experiencias de la práctica y acciones asociadas a la sistematización.

\section{Palabras clave:}

estudio de caso, investigación, necesidades de investigación, formación de profesores y práctica pedagógica.

\section{Necessities in research training for practicing teachers}

\section{Abstract}

Research about practice improves the professional development teachers' reflection, it contributes elements to built professional knowledge and allows setting practice communities. These communities are interested on teaching-learning process of mathematics as well as on practice as element to be problematized. Thus, the research training becomes a tool for thinking and changing the scholar educative practices and for building professional knowledge. Eight case studies were done as previous inquiry for designing a training proposal in research for inservice math teachers. In this inquiry, training needs of research were identified 
through non-participant observation and interviews. Also, the resources are teachers, their partners and principals. The meaning needs detected are associated with teamwork's dynamics, the experiences of practice socialization and actions related systematization.

\section{Keywords:}

Case study, research, research needs, teachers training and pedagogical practice.

\section{Necessidades de formação em pesquisa de professores em exercício}

\section{Resumo}

Pesquisar a prática promove a reflexão sobre o exercício profissional dos professores, fornece elementos para construir conhecimento profissional, oferece a possibilidade de constituir comunidades interessadas em problematizar a prática e os processos de ensino-aprendizagem das matemáticas. Assim, a formação em investigação torna-se numa ferramenta para pensar e transformar as práticas educativas e construir conhecimento profissional docente. Como entrada para projetar uma proposta de formação em investigação para professores de matemática em exercício, foi realizado um estudo no qual, se identificaram necessidades de formação em investigação através de oito estudos de caso, usando a observação no participante e a entrevista como instrumentos de coleta de informação e considerando como outras fontes os colegas, os diretivos e coordenadores. As principais necessidades identificadas são associadas com as dinâmicas de trabalho em grupo, a socialização das experiências da pratica e ações de sistematização das mesmas.

\section{Palavras-chave:}

Estudo de caso, pesquisa, necessidades de formação, formação de professores, pratica docente. 


\section{Introducción}

En el surgimiento de la relación docencia-investigación en Colombia, se identifica que desde 1986, los profesores de educación básica y media han determinado la necesidad de generar dinámicas de reflexión, que los estimulen a adoptarlas en su profesión como un factor de transformación de su práctica; de esta manera se han aislado de la idea de investigación positivista que fue enaltecida por profesionales externos y se dio inicio a un trabajo libre en sistematización de la propia práctica, involucrando a la práctica profesional algunas acciones del hacer investigativo.

Reconociendo tal relación y asumiendo la investigación como una acción involucrada con el hacer del aula, motor de construcción de conocimiento profesional y de transformación de la práctica (Perafán y Aduríz-Bravo, 2005), en el proyecto "Formación en y hacia la investigación para profesores de matemáticas en ejercicio", se asume la importancia de responder a las dinámicas de dicha relación, generando una propuesta de formación en investigación para profesores de matemáticas de educación básica y media que sin estudios de postgrado e interesados en sus prácticas, requieren cualificar sus procesos de reflexión e ir más allá de un nivel de descripción de su práctica (Piedra, Hernández y Rodríguez, 2013).

Siguiendo a Porlán, Martín del Pozo, Martín y Rivero (2001), una propuesta de formación debe estar fundamentada en los requerimientos de la población a la cual va dirigida. Así que para diseñar la propuesta de formación en investigación mencionada, se realizó un estudio de campo con el objetivo de detectar necesidades de formación en investigación de profesores de matemáticas de educación básica y media que laboran en Bogotá. En este artículo se reportan los resultados más importantes de dicho estudio.

\section{Referentes conceptuales}

Para realizar el estudio de campo fue imperativa una conceptualización del término necesidad asociado a la formación en investigación para un profesor, así como una configuración de ideas que permitieran detectar las necesidades desde diferentes perspectivas.

\section{Conceptualización del término necesidad}


Siguiendo a Tejedor (1990, citado en González, 2010), al Colectivo IOE (1998, citado en Suau, 2007), Maslow (2006, citado en Elizalde, Martí y Martínez, 2006) y Escamilla (2006, citado en González, 2010), la necesidad puede enmarcarse en diferentes contextos: desde la fisiología y las ciencias económicas, hasta las ciencias sociales y humanas. Particularmente, la necesidad puede caracterizarse como la carencia de algo que se considere deseable (colectivo IOE, 1998), también como una discrepancia medida entre el estado actual y el deseado (Beatty, 1981, citado en González, 2010). No obstante, Atkinson (1958, citado en Elizalde, Martí y Martínez, 2006) asoció el término necesidad a la disposición de buscar una finalidad o propósito, por lo que la necesidad se relaciona con el tránsito a cumplir con tal propósito.

A partir de las anteriores consideraciones, en esta investigación se define la necesidad como la diferencia entre un estado actual y un estado deseado o de deber ser, o aquello que permite el tránsito a un estado deseado o de deber ser (Sánchez, et al., 2012, p. 2.165).

\section{Miradas para la detección de necesidades}

Bradshaw (1972, citado en Suau, 2007) propone para el estudio de las necesidades humanas, clasificarlas de acuerdo al diagnóstico de los problemas de una comunidad, ya que no es suficiente con obtener información sobre su realidad social, se debe además, realizar juicios de valor basados en la opinión mayoritaria de sus miembros en contraste con la opinión de expertos. En este sentido, Bradshaw (1972, citado en Suau, 2007) distingue cuatro tipos fundamentales de necesidades:

1. Percibidas: aquellas necesidades distinguidas y sugeridas por la mayoría de los miembros de la comunidad.

2. Normativas: son las necesidades establecidas según el criterio de expertos, que emplean sus conocimientos técnicos y profesionales para hacer un juicio sobre la situación de la comunidad. En este tipo, también se encuentran las necesidades establecidas por personas que por su posición o función en la comunidad, disponen de información valiosa sobre su realidad.

3. Expresadas: son establecidas ante la identificación de comportamientos o situaciones problemáticas en la comunidad. 
4. Comparadas: son aquellas establecidas por comparación entre comunidades equivalentes o con características similares, para transferir necesidades de una a otra, o al tomar una como punto de referencia y comparación para la otra.

Puede notarse que las necesidades normativas y percibidas son expresadas por personas que conocen la comunidad y cuyo criterio es útil para la detección de sus necesidades; sin embargo, el criterio del investigador que se incorpora en la comunidad para conocerla, no se considera fuente para identificar otras necesidades. No obstante, se propone que esta también sea asumida para la detección y clasificación de necesidades. Así es que se establece un criterio para detectar y clasificar las necesidades, y es desde la persona que percibe la necesidad.

En este orden de ideas, las personas que pueden identificar necesidades de formación en investigación de una persona (en este caso el profesor de matemáticas en ejercicio) son: el mismo profesor, sus compañeros o pares, sus superiores (coordinadores o rector) y el investigador. Cada uno de ellos puede identificar y expresar necesidades al respecto de diferentes aspectos de la formación o actividad del docente observado. Más específicamente, las miradas para la detección de necesidades de formación en investigación que se propusieron para esta investigación son:

5. Desde el profesor: parte de lo que el profesor concibe como investigación, de lo que según él, debe caracterizar a un profesor para ser investigador de su propia práctica, en contraste con lo que reconoce tiene y aquello que le falta.

6. Desde sus pares: surgen del reconocimiento que los compañeros del profesor tienen acerca de las acciones que él desarrolla, y que podría reforzar para ser profesor investigador; esto, a partir de lo que ellos consideren sobre el hacer del profesor investigador de su propia práctica.

7. Desde un externo, cercano a su trabajo: se identifican a partir de lo que algún jefe inmediato (coordinadores o rector) distingue en el profesor, según lo que para él es investigación de la práctica.

8. Desde los expertos: asumido el grupo de investigación como expertos, en esta mirada se consideró la conceptualización de necesidad, las características del profesor que investiga su propia práctica y aquellas emergentes de los estudios de caso, asociadas al modelo teórico de dimensiones de la acción de investigar. Tal modelo se explicitará a medida que se muestren los resultados de los estudios. 
Dado que las necesidades de formación en investigación dependen de cada población, se realizó un estudio cualitativo con ocho estudios de caso y se establecieron necesidades de formación presentes en la mayoría de casos, siguiendo el camino metodológico que se expresa a continuación.

\section{Metodología}

Siguiendo a Bartolomé (1992), el estudio de caso es el método de investigación que permite comprender en mayor medida la sociedad y la realidad que se estudia, pues posibilita conocer a profundidad el contexto y la dinámica de los objetos estudiados. Así, el estudio de caso se constituye en el método utilizado para la identificación de necesidades de formación en investigación, siendo la observación y la entrevista las técnicas de recolección de información consideradas.

En el trabajo de campo se realizaron ocho estudios de caso a profesores de matemáticas en ejercicio de diferentes localidades de Bogotá (Colombia), quienes pertenecían a instituciones educativas públicas o privadas.

Se partió de la premisa que para realizar investigación sobre la práctica, lo primero que se requiere es interés sobre la misma; también que el ejercicio de haber realizado una experiencia significativa era muestra de tal preocupación. Así, la selección de los casos se realizó a partir de un estudio muestral en el que se ubicaron profesores de matemáticas de Bogotá, que hubiesen divulgado alguna experiencia significativa y que no tuvieran formación en investigación

Como resultado de la selección de los casos, se encontraron siete profesores de matemáticas de Bogotá que sin tener formación postgradual de maestría, ni alguna formación en investigación o algún tipo de participación en un proyecto de investigación, hubiesen realizado experiencias significativas. El octavo profesor con quien se completó el grupo, no tenía alguna experiencia significativa, pero se mostró interesado en conocer aspectos importantes de su práctica y aceptó formar parte del estudio.

Con cada profesor se hizo observaciones no participantes de su actividad profesional durante un tiempo aproximado de dos meses; el medio de registro principal fueron los diarios de campo y, cuando las instituciones lo permitían, el video de la clase. Adicionalmente, se aplicaron dos entrevistas semiestructuradas con cada profesor, la primera antes de iniciar la observación y la segunda luego de terminarla; estas entrevistas fueron registradas por medio de videos audiovisuales. 
Cada observación y entrevista estaba a cargo de un pasante de investigación que a su vez era estudiante para profesor de matemáticas de la Licenciatura en Educación Básica con Énfasis en Matemáticas (LEBEM) de la Universidad Distrital Francisco José de Caldas. Cada pasante acompañaba permanentemente a un profesor en los diferentes espacios de su práctica pedagógica (clases, reuniones de área, descansos, etc.) y además de diligenciar el diario de campo con las observaciones, conversaba con el profesor para indagar por aquellos aspectos problemáticos de su práctica, que no podían detectarse en la observación o evidenciarse en la realización de alguna entrevista.

El análisis profundo de las observaciones, las entrevistas y las conversaciones con los profesores, permitió obtener una clara idea del desarrollo de sus prácticas y de los elementos que permitirían el paso de dichos profesores, a ser investigadores de su propia práctica. A continuación se encuentra una descripción de los casos estudiados, denominando cada caso sin explicitar el género:

Perfil de los profesores

Profesor 1: licenciado en Matemáticas, con experiencia de tres años en colegios privados de Bogotá como docente de educación básica secundaria (grados: 6-9). En el momento del estudio, laboraba en un colegio con estudiantes de estratos 3 y 4 , ubicado en la localidad Antonio Nariño.

Profesor 2: licenciado en Matemáticas y especialista en Computación para la Docencia, tiene 25 años de experiencia profesional. Al momento del estudio, trabajaba como docente en un colegio privado estrato 4 ubicado en la localidad de Teusaquillo en Bogotá, y en un colegio público en la localidad Rafael Uribe Uribe de la misma ciudad, siendo en la primera institución donde se realizó el estudio. Es profesor de educación básica secundaria (grados: 6-9) y coautor de una serie de libros para la enseñanza de las matemáticas escolares. Ha escrito algunos documentos relacionados con actividades para enseñar matemáticas usando software educativo.

Profesor 3: licenciado en Educación Básica con Énfasis en Matemáticas, especialista en Lúdica y Recreación. Su experiencia es de 33 años trabajando en básica primaria, particularmente con estudiantes de 3, 4 y 5 grado. Al momento de realizar el estudio laboraba en una institución educativa distrital de Bogotá, ubicada en la localidad de Kennedy.

Profesor 4: licenciado en Matemáticas con 8 años de experiencia docente trabajando en educación básica secundaria y media. Al momento del estudio, laboraba en una institución educativa distrital de Bogotá, ubicada en la localidad de Usme. 
Perfil de los profesores

Profesor 5: licenciado en Matemáticas. Durante sus 3 años de experiencia, ha trabajado en un colegio privado de la localidad de Puente Aranda, enseñando matemáticas en básica secundaria y media.

Profesor 6: licenciado en Matemáticas. En sus 16 años de experiencia, ha trabajado en colegios privados y públicos, al momento del estudio se desempeñaba como profesor de Matemáticas en la educación básica secundaria en una institución educativa distrital ubicada en la localidad Rafael Uribe Uribe, Bogotá.

Profesor 7: licenciado en Matemáticas y Computación con 15 años de experiencia. Al momento del estudio trabajaba en una institución educativa distrital en la localidad de Fontibón en Bogotá, enseñando en los grados de 8 a 11.

Profesor 8: licenciado en Matemáticas. En sus 3 años de experiencia ha trabajado en colegios públicos y privados de Bogotá, enseñando Matemáticas en educación básica secundaria y media. Al momento del estudio, laboraba en una institución educativa distrital de la localidad San Cristóbal de Bogotá, y en un colegio privado de la localidad Rafael Uribe Uribe.

Para identificar necesidades de formación en investigación, en el caso de los profesores, se acudió a entrevistas, a observaciones no participantes y conversaciones informales; en el caso de los pares y los directivos, a una entrevista semiestructurada.

Las entrevistas realizadas al profesor tenían dos intenciones: la primera de ellas, era conocer algunos elementos de la práctica del profesor y profundizar en aquellos cuestionamientos que quedaron abiertos desde las observaciones; la segunda, era identificar lo que el profesor consideraba por investigación y los elementos que según él o ella debían fortalecer para ser un profesor investigador de la propia práctica. Cada intención determinaba un grupo de preguntas durante la misma sesión de entrevista realizada.

\section{Resultados}

Teniendo en cuenta que las necesidades de formación en investigación se establecieron a partir de las necesidades identificadas desde cuatro miradas distintas; los resultados se estructuran de acuerdo a cada mirada y en las conclusiones se establecen las necesidades de formación de los profesores a partir de la consolidación de lo presentado en los resultados. 


\section{Necesidades de formación en investigación establecidas desde los investigadores}

De acuerdo a la definición asumida de necesidad, era imperioso establecer un ideal de profesor investigador; por lo que el grupo analizó de manera teórica las acciones y actitudes que se develan cuando se investiga la propia práctica. A continuación se describirá brevemente las consideraciones conceptuales en dicho ideal.

La acción de investigar comporta el movimiento en tres dimensiones interrelacionadas entre sí, la dimensión personal por cuanto quien investiga es un sujeto que se cuestiona y cuyos hallazgos favorecen su formación; la dimensión social porque la acción de investigar se genera y valida en una comunidad, y es a ella a quien deben favorecer los resultados y aportes de la investigación; y finalmente, la dimensión realizativa porque dicha acción no es ingenua, tiene intención, y esta trae consigo el ejercicio de diversas actuaciones que le dan vida a la acción de investigar.

De cada dimensión se despliegan algunas características que corresponden a conocimientos, acciones y procedimientos de la acción investigativa. De esta forma, se encuentran la crítica y la problematización asociada principalmente a la dimensión personal, la interacción a la dimensión social y la estrategia a la realizativa. A su vez, cada característica se encuentra constituida por subcaracterísticas que en conjunto permiten conceptualizarla y que comportaron las categorías a analizar durante los estudios de caso.

Los resultados de las necesidades de formación en investigación detectadas desde la mirada de los investigadores, se presentarán siguiendo las características generales (crítica, problematización, interacción y estrategia) y se estructuraron con base en las subcaracterísticas que las conforman, y que se irán señalando al tiempo que surgen en la descripción de las necesidades identificadas.

Crítica: se entiende la crítica como "la actitud permanente por reconocer y cuestionar elementos o situaciones de la práctica profesional (...)" (Sánchez, et al., 2012, p. 2.167). Se encuentra constituida por las subcaracterísticas: reflexión, apertura al cambio, búsqueda de verdad, ser analítico y ser social.

Se estableció que la reflexión es permanente en cada uno de los profesores, sin embargo, en algunos de ellos es necesario que la práctica se convierta en un elemento de asombro, para que de ella se identifiquen aspectos conflictivos, de cuya problematización derive la mejoría en la práctica; esto también fomenta el desarrollo de actitudes como el ser inquisitivo, pues se identificó que esta 
subcaracterística está ausente, debido a la no problematización del hacer en el aula.

Asimismo, la apertura al cambio es una característica difícil de alcanzar, pues es natural la negación al rompimiento de esquemas asumidos, aún más cuando se considera que estos permiten lograr a cabalidad lo que se propone; al respecto, se identificó en tres casos que la participación en eventos, la documentación y la interacción con los pares se constituyen en herramientas importantes que reflejan la insuficiencia de mantener estáticas ciertas dinámicas.

Otros aspectos como la búsqueda de la verdad, el ser analítico o social, se asocian principalmente con lo que se considera de la clase de matemáticas, de las variables que confluyen y que deben considerarse, para desarrollar la práctica educativa. En general, los profesores confían que la argumentación es una herramienta fundamental para construir conocimiento, sin embargo, esta acción generalmente recae sobre el profesor, la búsqueda de información se centra principalmente en el objeto matemático a enseñar únicamente y a pesar de que existe claramente la conciencia del papel social del profesor de matemáticas, dicha conciencia tiene poca influencia al interior del aula.

Estrategia: se asume como "la acción de planificar y orientar su actuar hacia la solución de problemas de su hacer cuando el hacer está en investigación" (Sánchez, et al., 2012, p. 2.169) y se encuentra constituida por las subcaracterísticas, ser perseverante, conocer métodos de investigación, sistematizar, establecer metas y ser creativo.

Como necesidad de formación se encuentra el conocimiento sobre investigación (por ejemplo, métodos de investigación y formas de sistematizar). Además, características como la perseverancia y la creatividad se ponen en juego no solo en el accionar de actividades para enseñar matemáticas, sino también en aquellos momentos donde permea las situaciones operativas del sistema educativo, con la defensa y puesta en marcha de propuestas nuevas que incluyan el uso de diversas herramientas. Particularmente, estas dos características (perseverancia y creatividad) como necesidades se hacen visibles ante la imposibilidad que en muchas ocasiones se presenta, de llevar al aula, aquellas propuestas que favorecen la generación de nuevos ambientes de aprendizaje y que pueden llevar al docente por un camino de mejora de su práctica.

Adicionalmente, se identificó que los profesores establecen metas y generan estrategias para conseguirlas; no obstante, dichas metas y estrategias responden a intenciones netamente cognitivas, es decir, se refieren al conocimiento que se desea que los estudiantes construyan. Determinar otro tipo de metas depende 
principalmente de lo que el profesor considere como importante a desarrollar en su práctica pedagógica, y por ende, es necesario ampliar la mirada sobre la misma y potenciar el reconocimiento de que existen otro tipo de propósitos que también son importantes en la práctica y que deben ser abordados.

Finalmente, sistematizar es una de las características en las que más se requiere desarrollo para formar un profesor investigador; de los casos se deduce que son pocos los momentos en los que los profesores toman apuntes de sus clases, y cuando lo hacen dichos registros no son insumo para mejorar su práctica. Considerando algunas de las respuestas obtenidas en las entrevistas, los profesores expresan que esta actividad requiere tiempo y disposición de escritura, actividad que no es atrayente para el profesor que a diario tiene diversas clases.

Interacción: se entiende como "la actitud permanente de relacionarse con otros para construir conocimiento profesional que trascienda" (Sánchez, et al., 2012, p. 2.169) y la constituyen las subcaracterísticas trabajar en equipo, socializar, construir colaborativamente e incentivar la investigación.

Se identificó que los profesores pueden trabajar en equipo, construir colaborativamente conocimiento, y en algunas ocasiones intentan socializar sus experiencias, o escuchar las de los demás, en favor de construir soluciones a sus problemáticas o en favorecer la solución de los problemas de la actividad profesional de sus compañeros. Sin embargo, las condiciones institucionales desestimulan o no favorecen las acciones de interacción entre pares de la institución, como reuniones de área, conformación de grupos de trabajo o actividades académicas en estos grupos, de modo que los profesores terminan trabajando individualmente o simplemente abandonando las tareas propuestas en relación con sus prácticas; así mismo, el interés por interactuar entre pares para resolver los problemas de sus prácticas 0 para realizar proyectos académicos de reflexión, investigación o innovación, no es un interés compartido por todo el equipo de docentes, lo que agrava aún más el problema de los profesores que desean reflexionar o investigar sus prácticas, pues terminan aislados y trabajando por separado, buscando en algunas ocasiones, de manera individual, soluciones a problemas que se les presentan. Así, es necesario que los profesores pertenezcan a equipos de trabajo con profesores que tengan sus mismos intereses y motivaciones.

Problematización: se entiende como "el proceso de identificar elementos en una situación, que le permitan plantear un problema de investigación, partiendo de una tensión entre lo que sucede y lo que el profesor sabe al respecto" (Sánchez, et al., 2012, p. 2.169). Está constituida por las subcaracterísticas documentarse de 
la práctica, tener capacidad de asombro, reconocer en su práctica situaciones o aspectos de interés, conflicto o tensión, documentarse sobre la práctica e identificar problemas de investigación.

Se detectó que los profesores se preocupan por identificar en sus estudiantes errores y dificultades, así como por proponer situaciones o actividades que favorezcan el aprendizaje de las matemáticas. Estas resultan ser el tipo de situaciones que representan conflicto o tensión en sus actividades de aula, en algunas ocasiones, se identificó que otras cuestiones como la disciplina, el interés, los factores psicológicos, motivacionales, emocionales o personales también interesan a los profesores.

A pesar de que se identificó que los profesores se documentan de su práctica al usar actividades previamente aplicadas, reconocer dificultades de sus clases y considerar manifestaciones de los estudiantes como insumo para evaluar algunos aspectos de su desarrollo profesional; la falta de documentación sobre la práctica impide la articulación de la teoría y la práctica, que en este caso constituye la relación entre la teoría y los problemas o situaciones de aula identificadas por el profesor. Adicionalmente, tales situaciones no son vinculadas con los cuerpos de conocimiento ya existente, para identificar problemas de investigación. En las subcaracterísticas de la problematización, se encuentra el documentarse de la práctica y documentarse sobre la práctica. Aunque el nombre es similar, se diferencian en que la primera hace referencia al asumir la práctica como insumo para inquietarse o reflexionar sobre ella, mientras que la segunda, a la búsqueda de información sobre algunas variables o aspectos que forman parte de la misma.

\section{Necesidades identificadas desde el profesor}

Para la identificación de necesidades desde el profesor, se aplicaron entrevistas que indagaban por su noción de investigación, las características que él considera debe tener un profesor investigador y aquellas que según él, tiene y las que necesita. Cabe aclarar que tales características no necesariamente coinciden con la caracterización de profesor investigador que el grupo de investigación planteó, pues varían en cada caso, y por ello las necesidades expuestas por los profesores están basadas en su consideración.

Respecto a la noción de investigación, los profesores la conciben principalmente como un proceso; dos de ellos la entienden como un método o estudio, para referirse a que esta de alguna manera trae un riguroso modo de proceder. 
La mayoría de los profesores expresan que la investigación permite la solución de problemas de la práctica docente, y solo tres profesores destacan la función de la investigación en la producción de conocimientos, más allá de resolver algún problema de su práctica. Finalmente, buena parte de los profesores reconocen algunos momentos del proceso investigativo, como la documentación, la aplicación de instrumentos y el análisis de datos.

Algunas de las características expresadas por los profesores en los estudios de caso se asociaron, según su coincidencia, con las características del profesor investigador determinadas por el grupo de investigación. Estas características fueron:

- La curiosidad, que se encuentra asociada a la característica de ser inquisitivo de la crítica.

- Ser innovador, que puede ubicarse en la subcaracterística ser creativo, perteneciente a la estrategia.

- Sistematizar información, ya sea para registrar hechos de la clase o para analizar información recolectada en el proceso de la investigación, por lo que se asocia con la subcaracterística de sistematizar en la estrategia.

- Leer permanentemente sobre avances e innovaciones relacionados con los procesos de enseñanza que el profesor diseña e implementa en su actividad docente, que puede relacionarse con la subcaracterística de documentarse sobre la práctica en la problematización.

- La habilidad de escribir textos para producir documentos que divulguen los resultados obtenidos en las investigaciones, que se relaciona con el indicador tiene o elabora algunos escritos que considera importantes acerca de lo que lee o de lo que sucede en su práctica, de la subcaracterística sistematizar en la estrategia. En este punto es importante especificar que asociado a cada subcaracterística, se propusieron indicadores que hacían referencia a aquellos indicios de existencia de la subcaracterística.

- El liderazgo, asociado a la perseverancia, que hace referencia a guiar y ejecutar el proceso hasta cumplir sus objetivos o resolver los problemas que se propuso.

- Tener habilidad para la elección de problemáticas para investigar, que se encuentra relacionada con la subcaracterística identificar problemas de investigación en la problematización. 
Solo dos profesores expresan la característica de interacción con los pares u otros miembros de la comunidad escolar y académica para el desarrollo de la investigación, esta se asocia con la característica de interacción propuesta por el grupo de investigación.

Otras características que mencionaron los profesores fueron: la voluntad para hacer investigación, que de hecho se corresponde con la definición de actitud considerada en esta investigación; la vocación docente, que lleva al docente a querer mejorar sus prácticas; conocer métodos para resolver problemas y conocer teorías para orientar la investigación.

Finalmente, las principales necesidades enunciadas por los profesores se refieren a la sistematización, la documentación permanente sobre la práctica y la escritura de textos para la divulgación de los resultados. Solo un profesor expresó que necesitaba conocer métodos y teorías para orientar la investigación o resolver problemas de su práctica.

\section{Necesidades identificadas desde el par y directivos}

A partir del análisis conjunto de las entrevistas, específicamente de las preguntas diseñadas para identificar la concepción de investigación descrita por los pares y directivos, con el fin de vislumbrar el marco de ideas de investigación a partir del cual ellos expresaron las necesidades de formación, se encontró que:

- Coinciden en cinco de los estudios de caso, al considerar la investigación como la acción de consultar información o el proceso que inicia con la búsqueda de esta, además de indicar que el propósito de dicha indagación es mejorar las prácticas en el aula de clase considerando, por ejemplo, que la realidad es dinámica (los estudiantes cambian, la sociedad cambia y los contextos cambian). Desde las diferentes entrevistas, los pares y directivos señalan como medio para la consulta o búsqueda de información, la asistencia a seminarios y la búsqueda de textos.

- En dos de los estudios de caso, la concepción de investigación se centra en la capacidad del profesor para ver, analizar, manejar y cambiar sus prácticas pedagógicas.

- Finalmente, en uno de los estudios de caso se manifiesta que la investigación es un proceso en el que existen una serie de pasos cíclicos que inician con la formulación de una hipótesis. 
En consecuencia con las concepciones anteriormente expuestas, directivos y pares señalan el quehacer deseable de un profesor de matemáticas investigador de su propia práctica, a través de acciones específicas en su gestión en el aula, el trabajo con colegas y la divulgación de sus propuestas de clase; dicho hallazgo permitió establecer que las acciones atribuidas al profesor investigador de su propia práctica por los pares y directivos confluyen en algunas de las subcaracterísticas propuestas por el grupo de investigación, razón por la cual es posible presentar a partir de dichas coincidencias las necesidades de formación en investigación.

En general, los pares y directivos expresan las necesidades de sus compañeros aludiendo a los escenarios comunes con él o ella en la vida escolar, siendo estos principalmente la reunión de área y eventos escolares; en consecuencia, entre las necesidades señaladas se prioriza el ser propositivo, entendiendo este término como una actuación crítica y creativa espontánea, caracterizada por plantear y ejecutar opciones o alternativas de solución a los problemas o situaciones existentes; idea que, según el caso del profesor al que se refiera, está discriminada por niveles que van desde tomar la iniciativa de formular y ejecutar ideas, hasta considerar en algunos casos que las acciones y propuestas del profesor deberían ser socializadas a nivel micro -en el colegio, con los compañeros de área- y a nivel macro -en eventos académicos-.

En el primer nivel de lo denominado ser propositivo, para dos de los pares y directivos entrevistados, es indispensable que los docentes tomen la iniciativa de formular una intención o proyecto y a partir de este, hacer uso de recursos, mecanismos o instrumentos para su ejecución; idea ligada con el establecer metas y ser metódico, que bajo el planteamiento del grupo corresponde a subcaracterísticas asociadas a la estrategia.

Continuando con la idea anterior, se expresa que la intervención del docente a partir de sus ideas, intenciones o proyectos debe partir de argumentos sólidos, que le permitan explicar cada una de sus posiciones y el porqué de ellas, además de buscar permanentemente explicaciones válidas a lo que dice, hace y piensa. Esta necesidad reposa en la idea expresada en confiar en el razonamiento asociado a la crítica, ya que se establece el proceso argumentativo como el mecanismo para consolidar planteamientos y sustentar ideas en los escenarios en los que el profesor actúa.

En un segundo nivel del ser propositivo, cuatro de los pares y directivos entrevistados reconocen en sus colegas la formulación, el desarrollo y la puesta en escena de ideas en la ejecución de sus clases, eventos escolares y reuniones de área. En consideración a dicho reconocimiento, para los colegas y directivos resulta 
primordial que los docentes realicen algunas acciones que beneficien no solo su acción individual sino el quehacer de su grupo de compañeros; en este sentido, por ejemplo, si el profesor solo pone en práctica sus ideas para el desarrollo de su clase, valdría la pena exponer sus ideas o proyectos en las reuniones de área, incentivando el debate para la creación y evaluación de las mismas con el ánimo de potenciar cambios en los procesos de enseñanza-aprendizaje institucionales. Dicha perspectiva coincide con lo denominado por el grupo como socializar con los pares a partir de los espacios institucionales que posibilitan dicha interacción.

Continuando con la idea de socializar con los pares, en uno de los casos el docente manifiesta que existe una gran intervención y participación del profesor con proyectos e ideas a nivel institucional, razón por la cual no solo sugiere como necesario que dé a conocer sus ideas de intervención en el aula con compañeros de trabajo sino en eventos académicos, lo que le posibilitaría presentar sus trabajos a otros profesores. A partir de dicha recomendación se infiere la necesidad de compartir sus experiencias con una comunidad externa a la institución, posiblemente en eventos académicos, idea que se encuentra asociada a la característica denominada por el grupo como interacción.

Finalmente, a la luz de la coincidencia de las características, las principales necesidades señaladas por pares y directivos, se centran en la dimensión social, en las actitudes y acciones asociadas a trabajar en equipo, construir colaborativamente y socializar con los pares y en eventos académicos.

\section{Conclusiones}

En diversas ocasiones los procesos asociados a la investigación de la propia práctica no se desarrollan debido a la concepción que tienen algunos profesores de que la investigación y la práctica son dos acciones no relacionadas, además porque se concibe que la investigación requiere de conocimientos superiores y de tiempos especiales, distintos a los destinados al ejercicio de la práctica docente. De hecho, el trabajo comunado con profesores universitarios fomenta el desarrollo de actitudes y conocimientos propios de investigación, así mismo, propicia que los profesores piensen sobre sus prácticas y cuestionen aspectos que antes eran incuestionables para ellos.

Por otro lado, la interacción con pares y el espacio para realizar cuestiones relacionadas con sus prácticas diferentes al trabajo de aula, es fundamental para el desarrollo de actitudes propias de investigación. Proporcionando a los profesores nuevas 
miradas sobre sus prácticas. De hecho, la participación en eventos es un insumo importante para reconocer otras formas de enseñar, nuevas herramientas a utilizar e inclusive, al incorporar nuevos elementos en las clases de matemáticas. Allí, se puede identificar que de distintas propuestas se obtienen resultados satisfactorios, esto puede fortalecer la confianza en aplicar propuestas diferentes en el aula de clases.

Desde los estudios de caso y teniendo en cuenta las miradas del profesor, los investigadores, los directivos y pares, se concluye que existe coincidencia al considerar que acciones como la escritura y la lectura se constituyen como necesidades para investigar, así como la interacción con pares ya sea para dar a conocer sus experiencias, o para intercambiar impresiones u aportes de las situaciones de los otros.

Particularmente, las principales necesidades de formación en investigación expresadas por los profesores son documentación permanente sobre los problemas de su práctica, la sistematización de datos recolectados de experiencias significativas y la consecuente producción de escritura de textos de divulgación de resultados. Es interesante que solo un profesor haya considerado como necesidad el estudio de métodos y teorías para orientar la investigación o resolver problemas de su práctica.

Por su parte, para los directivos y pares, las principales necesidades de formación en investigación de la práctica se encuentran asociadas con la característica de interacción, principalmente en lo relacionado con la socialización de las experiencias de los profesores a sus pares en la institución y en eventos académicos, de modo que las acciones del profesor incentiven a otros profesores, potencien los cambios en los procesos de enseñanza-aprendizaje en diferentes instituciones y se promueva el intercambio de ideas que enriquezcan la labor del profesor investigador. Otra necesidad expresada por directivos y pares se encuentra relacionada con el uso del proceso argumentativo como mecanismo para consolidar planteamientos y sustentar ideas en los escenarios en los que actúa el profesor.

Adicionalmente, las principales necesidades de formación en investigación detectadas por los investigadores son: la documentación sobre la práctica para articular la teoría y la práctica; convertir la práctica en un elemento de asombro, de tal forma que se identifiquen aspectos conflictivos, de cuya problematización deriven cambios y mejora de la práctica y la comprensión de los fenómenos del aula; ser inquisitivo y abierto al cambio; la sistematización, pues las actividades asociadas a esta requieren de tiempo y disposición de escritura y, ante la gran cantidad de actividades que desarrollan los profesores a diario junto con la dificultad para escribir, resulta ser una acción poco atractiva. 
Aunque los directivos y pares consideran al trabajo en equipo como una de las principales necesidades de formación para los profesores, los investigadores detectan que en las instituciones educativas no siempre se estimulan o favorecen estas acciones, incluso no todos los profesores se encuentran interesados en desarrollar actividades colectivas de reflexión, investigación o innovación, lo que hace que los profesores interesados terminen individualmente solucionando los problemas que se les presentan. Por lo anterior, se reconoce la necesidad de vincular a los profesores a equipos de trabajo con otros profesores que tengan sus mismos intereses y motivaciones.

En lo que respecta a la formación en investigación para profesores, se requiere como primera medida el desarrollo de actitudes asociadas con el cuestionamiento, el asombro, la indagación y la realización. Una propuesta alejada de los intereses de los profesores o de sus prácticas en particular, tiende a no tener incidencia real en las prácticas, pues los profesores incorporan en sus clases solo aquello que sienten propio y benéfico para su principal propósito: el aprendizaje de los estudiantes.

Adicionalmente, una propuesta de formación en investigación debe realizarse por medio del acompañamiento permanente a los profesores, deben proponerse actividades que partan de su práctica, y adicionalmente las acciones que generen conocimiento deben estar permanentemente acompañadas y complementadas, pues en los estudios se observó que el trabajo permanente que realiza uno de los profesores con un profesor universitario, ha tenido incidencias tanto en lo que él concibe de su práctica, como en la intención de realizar acciones de investigación de su propia práctica.

\section{Referencias}

Bartolomé, M. (1992). Investigación cualitativa en educación: ¿comprender o transformar? Revista de investigación educativa, 20(2), 7-36.

Cruz, M. (2007). La formación del docente reflexivo. Recuperado de http://actualizacion. sev.gob.mx/pdf/el_docente_reflexivo.pdf

Elizalde, A., Martí, M. y Martínez, F. (2006). Una revisión crítica del debate sobre las necesidades humanas desde el Enfoque Centrado en la Persona. POLIS, revista de la Universidad Bolivariana, 15(5). Chile. Recuperado de http://istas.ccoo.es/descargas/ Revisi\%C3\%B3n\%20cr\%C3\%ADtica\%20de\%20las\%20necesidades.pdf 
González, B. (2010). Perfil profesional y necesidades de formación del docente especialista en dificultades de aprendizaje. (Tesis doctoral). Universidad Rovira I Virgili. Recuperado de http://tesisenred.net/handle/10803/42932

Piedra, D., Hernández, E. y Rodríguez, J. (2013). El estado de la reflexión sobre la práctica de aula. Una muestra por conveniencia de profesores de matemáticas en Bogotá. Acta Latinoamericana de Matemática Educativa, 26, 1.505-1.512.

Porlán, R., Martín del Pozo, R., Martín, J. y Rivero, A. (2001). La relación teoría-práctica en la formación permanente del profesorado. Sevilla: Díada.

Sánchez, B., Torres, J., Fonseca, J., Rodríguez, J., Piedra. P. y Hernández, E. (2012). Necesidades de formación en investigación y características del profesor investigador. Memorias del III Congreso Internacional y VIII Nacional de investigación en educación, pedagogía y formación docente.

Suau, J. (2007). Las voces de las personas y entidades implicadas en la educación como guía para la elaboración de propuestas en formación permanente del profesorado no universitario. (Tesis doctoral). Universidad de Lleida. Recuperado de http://www.tdx. cat/bitstream/handle/10803/8298/Tjsc1de1.pdf?sequence $=1$ 